\title{
An Investigation into Error Source Identification of Machine Tools Based on Time-Frequency Feature Extraction
}

\author{
Dongju Chen, Shuai Zhou, Lihua Dong, and Jinwei Fan \\ College of Mechanical Engineering \& Applied Electronics Technology, Beijing University of Technology, Beijing 100124, China \\ Correspondence should be addressed to Dongju Chen; dongjuchen@gmail.com
}

Received 19 June 2015; Revised 30 October 2015; Accepted 15 November 2015

Academic Editor: Toshiaki Natsuki

Copyright (C) 2016 Dongju Chen et al. This is an open access article distributed under the Creative Commons Attribution License, which permits unrestricted use, distribution, and reproduction in any medium, provided the original work is properly cited.

\begin{abstract}
This paper presents a new identification method to identify the main errors of the machine tool in time-frequency domain. The low- and high-frequency signals of the workpiece surface are decomposed based on the Daubechies wavelet transform. With power spectral density analysis, the main features of the high-frequency signal corresponding to the imbalance of the spindle system are extracted from the surface topography of the workpiece in the frequency domain. With the cross-correlation analysis method, the relationship between the guideway error of the machine tool and the low-frequency signal of the surface topography is calculated in the time domain.
\end{abstract}

\section{Introduction}

Machining accuracy is an important performance factor for machine tools, especially under circumstances in which relatively high precision is one of the basic requirements [1]. During the machining process, a variety of errors are in play at the same time, and this will be reflected in the surface topography of the workpiece with varying degrees. Errors of a machine tool include static errors and dynamic errors. Static errors are related to the structure of the machine tool, primarily caused by the geometric errors of the components of the machine tool; this part of the error can be identified from the experimental results by error shape. The dynamic errors of a machine tool are mainly the mechanical distortion of the workpiece and tool, the vibration of the structure of the machine tool, and the tracking error of the controller, which can introduce spatial frequency domain error; thus, this type of error should be identified from the frequency domain. The vibrations caused by the unbalance may destroy critical parts of the machine tool, such as bearings and couplings, which make up the major portion of the observed vibration frequency spectra of the machinery. These vibration spectra can be used to determine the error sources from the machine tool [2]. Before identification, the signal data should be processed first, and its main features should be extracted and determined.
Under certain machining conditions, the surface topography of the workpiece that turned by a machine tool is generated and then is measured through direct measurement of a profiler; thus, a fast and satisfactory identification method should be used. The premise of identification is processing the measured result first and extracting the important features in the signal. In conventional signal processing, the Fourier transform is a direct and commonly used method [3, 4]; a complicated high-order exponential function is used to transform the signal in the time domain into a function in the frequency domain, but the local signal cannot be well analyzed by this method. Local features can be described by the short-time Fourier transform [5], which appears subsequently, but the signal in the frequency domain cannot be localized in the time domain. For nonstationary signals, the wavelet transform is an effective method for processing the signal, which is an analysis method of a signal in the time and scale (frequency) domains. It is a multiresolution analysis method and is known as the microscopic representation of the signal. The biggest advantage is that, in the time and frequency domains, there is a good localized nature: the short time signal with high frequency can be localized, and there is also an accurate trend analysis for low-frequency signals, which provides a special advantage in terms of the measurement of the mutant signal and identification of the fault signal. Wavelet analysis in the field of manufacturing 
is applied in online monitoring of tool breakage and fault diagnosis of bearings and gears. Li et al. [6] researched the influence of machine vibrations on hard turned surface topographies based on the FFT and wavelet and analyzed the tool behaviour by spatial domain frequency analyses based on the fast Fourier transform, and wavelet reconstruction was used for profile filtering. Lingadurai and Shunmugam [7] proposed the metrological characteristics of the wavelet filter for processing of engineering surfaces and established a few typical manufactured surfaces and their reference surfaces by the wavelet filter and brought out the waviness content and phase matching by random process techniques. AntoninoDaviu et al. [8] studied the wavelet signals at different levels and applied the DWT to analyze numerical and experimental data for the diagnosis of dynamic eccentricities in induction motors. Chand [9] proposed SC- and SS-wavelet transforms that use the cosine and sine signals defined over the smaller intervals that help represent the Fourier transform of a signal in a better way. The SC- and SS-wavelet transforms provide not only sharper time-frequency localization but also much more information in a better localized form than the Awavelet transform; Zhu et al. [10] reviewed the state of the art of wavelet analysis for tool condition monitoring (TCM), introduced wavelet approaches, and discussed the superiorities of wavelet analysis to Fourier methods for TCM based on the nature of monitored signals. Bhowmik et al. [11] used an electromagnetic transient program (EMTP) to model a real transmission system and MATLAB for DWT and NN, and various types of faults were identified. The Fourier transform cannot be directly used for the signals which are random in the time domain because the signals have no integral condition. The power spectral density (PSD) and autocorrelation functions are coupled with the Fourier transform. PSD describes how the power of a signal or time series is distributed over the different frequencies. As a spectrum function, PSD has a natural advantage in the evaluation of the frequency domain. Bonte et al. [12] proposed a new formula to consider phase differences in the determination of an equivalent von Mises stress power spectral density (PSD) from multiple random inputs. Yoshida [13] analyzed the data of acceleration broadband with PSD; the signal was analyzed according to the height of the peak, and the power spectrum with the statistical properties is generally used as the basis of spectral analysis. Lu et al. [14] analyzed the random vibration signals of a vehicle's coupled longitudinal tracking system with PSD. Random vibration theory was used to obtain the response power spectral densities, by using PEM to transform this random multiexcitation problem into a deterministic harmonic excitation problem and then applying symplectic solution methodology.

With the provided analytical and numerical approaches, some researchers have studied the effects of the unbalanced parts on the machining process and the behaviour of the machine tools. Schulz and Würz [15] showed the systemspecific limitations of the component focused balancing and discussed the requirements by means of analytical and numerical approaches. The dynamic unbalance of the spindle is evaluated by a double-rotor model of spindledrawbar-bearing [16] and harmonic wavelet [17]. The fast
Fourier transform (FFT) is used to process the signal to reduce computation time [18] and satisfy magnitudewise shift invariance [19]. Recently, correlation analysis has been used in many research fields. Wieleba [20] used the correlation analysis in tribological research, evaluating the coefficient of friction and wear rate of PTFE composite with steel counterface roughness and hardness. Lockwood and Reynolds [21] presented a technique for automatically characterizing the three-dimensional geometry of fracture surfaces and used digital image correlation (DIC) to provide an accurate and fast method for digitally reconstructing fracture surfaces.

From the above, the previous work merely measured or modelled the single component error of machine tools; these methods will introduce some errors, and the analysis results are different from the actual values. Some researchers analyzed and identified the motion errors of machine tools from several measured results; however, the measured signal from the components of the machine tools cannot represent the actual processing surface. Therefore, the method which identifies the dominant error from the surface topography of the workpiece is more accurate.

In this paper, the surface topography of the workpiece is transformed into a low-frequency part and a high-frequency part by wavelet transform method. Then, the main features are extracted from the high-frequency signal part in the frequency domain by a power spectrum analysis method; the correlation degree of the geometric error of the machine tool and the low-frequency part of the surface topography is calculated in the time domain by the cross-correlation method. Finally, the experimental scheme for the vibration signal of the spindle system in different measurement conditions is designed, and the numerical analysis is verified.

\section{Analysis of the Surface Topography of the Workpiece}

2.1. Surface Topography of Workpiece Generation. In the experiment, a cylindrical aluminum alloy is selected as the processing part for machining. The machining machine tool is a vertical two-axis lathe in the laboratory, its frame is shown in Figure 1, and it has $X$ and $Z$ guideways. The workpiece (number 2) is supported by a hydrostatic spindle (number 1), and the lathe is used to turn the end face of the workpiece. The dimension of the end face includes a diameter of $20 \mathrm{~mm}$; maximum rotating spindle speeds of $150 \mathrm{rpm}$ and $110 \mathrm{rpm}$ are used in the processing. The upper end face of the cylinder workpiece is turned by the tool (number 5), which is driven by $X$ (number 3 ) and $Z$ guideways (number 4 ). The surface topography of the workpiece is measured by a profile testing instrument, and the type is PGI1240. The stylus moves along the radius from the centre of the workpiece to the edge. The displacement is $10 \mathrm{~mm}$ because the cylinder is symmetric; thus, the measured result shown in Figure 2 is approximately symmetric, and the horizontal coordinate, that is, the test range, is from $-10 \mathrm{~mm}$ to 0 and from 0 to $10 \mathrm{~mm}$. The result is a $2 \mathrm{D}$ image, and the ordinate coordinates represent the form and waviness of the surface. In Figure 2, the green curve shows the flatness and waviness of the surface turned by 


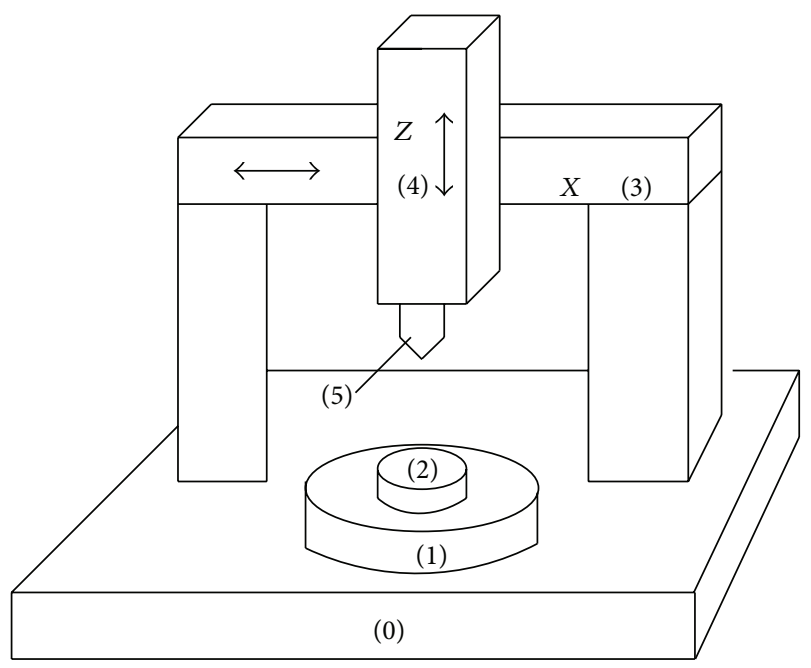
(0) Bed
(1) Spindle
(3) $X$ guideway
(2) Workpiece
(4) $Z$ guideway
(5) Tool

FIGURE 1: Scheme of the machine tool.

the machine tool in Figure 1. The red line expresses the standard centreline of the profile testing instrument, and the maximum form error is shown by the symbol $P_{z}$ or $P_{t}$ in Figure 2; the value is $2.0465 \mu \mathrm{m}$.

The surface topography of the part reflects the errors of the machine tool, which are equivalent to an integrated signal. The integrated signal includes many features and frequencies corresponding to the key components of the machine tool. It is difficult to extract a single error of the machine tool from the integrated signal directly; thus, extracting means and processing methods are needed. At first, the wavelet transform is used to decompose the integrated signal into different frequencies signal by a type of wavelet.

2.2. The Wavelet Transform. The wavelet method is a useful tool for processing signals. It was developed as an alternative to the short time Fourier transform (STFT) to overcome problems related to its frequency and time resolution properties. More specifically, unlike the STFT, which provides uniform time resolution for all frequencies, the DWT provides high time resolution and low-frequency resolution for high frequencies and high-frequency resolution and low time resolution for low frequencies. In that respect, it is similar to the human ear, which exhibits similar time-frequency resolution characteristics.

The inversion formula of the wavelet transform is

$$
f(t)=\frac{1}{C_{\psi}} \int_{0}^{+\infty} \int_{-\infty}^{+\infty} \frac{1}{a^{2}} W_{f}(a, b) \bar{\psi}_{a, b}(t) d b d a .
$$

This shows that the signal $f(t)$ can be reconstructed by the wavelet transform $\psi_{a, b}(t)$. Here, the parameters $(a, b)$ of the wavelet base functions $\psi_{a, b}(t)$ are restricted to some discrete points.
Discretization of Scale Parameter $a$. Usually $a=a_{0}^{j}$ and $j=0, \pm 1, \pm 2, \ldots, a_{0}>1$; the corresponding wavelet is

$$
\frac{1}{\sqrt{a_{0}^{j}}} \psi\left(\frac{t-b}{a_{0}^{j}}\right)=a_{0}^{-j / 2} \psi\left(a_{0}^{-j}(t-b)\right) .
$$

Discretization of Displacement Parameter $b$. For $j=0$, a suitable parameter $b_{0} \in R$ ( $R$ is real set) is considered, and the function $\psi\left(t-k b_{0}\right), k=0, \pm 1, \ldots$, can cover the entire time axis and the information will not be lost.

For other scales $a_{0}^{j}, j=0, \pm 1, \pm 2, \ldots$, the relative wavelet function $a_{0}^{-j / 2} \psi\left(a_{0}^{-j}(t-b)\right)$ because its width on the time axis is $a_{0}^{j}$ times the wavelet mother function $\psi(t)$, so that the sampling interval can be expanded by a factor of $a_{0}^{j}$ and will not cause information loss; the displacement parameter is then

$$
b=a_{0}^{j} b_{0} .
$$

The wavelet function that cannot lose the information after discretization is as follows:

$$
\frac{1}{\sqrt{a_{0}^{j}}} \psi\left(\frac{t-k a_{0}^{j} b_{0}}{a_{0}^{j}}\right)=a_{0}^{-j / 2} \psi\left(a_{0}^{-j} t-k b_{0}\right), \quad j, k \in Z .
$$

The time axis is adjusted, and the value of $k b_{0}$ is transformed into the integer $k$; the discrete wavelet function is then

$\psi_{j, k}(t)=a_{0}^{-j / 2} \psi\left(a_{0}^{-j} t-k\right), j, k \in Z$. Finally, the discrete wavelet transform of the signal $f(t)$ is

$$
W_{f}(j, k)=\left\langle f(t), \psi_{j, k}(t)\right\rangle=\int_{R} f(t) \bar{\psi}_{j, k}(t) d t .
$$

In the following, the $\mathrm{db} 1$ wavelet basis function is used to decompose the surface topography of the workpiece.

2.3. Processing of the Measured Signal. The measured result of the machined part is processed by the db1 wavelet, and the processed signal contains a low-frequency part and a highfrequency part, and each type of signal has ten layers of data.

Test data can be expressed by the function $x(t)$ :

$$
x(t)=\sum_{i=1}^{m} x_{i}(t)+\sum_{i=m+1}^{n} x_{i}(t)=s_{1}(t)+s_{2}(t) .
$$

The first part, $s_{1}(t)$, constitutes the low-frequency signal:

$$
s_{1}(t)=x_{1}(t)+x_{2}(t)+\cdots+x_{m}(t) .
$$
signal:

The second part, $s_{2}(t)$, constitutes the low-frequency

$$
s_{2}(t)=x(t)-s_{1}(t) .
$$

The signal $x(t)$ is converted through the wavelet transform in (6), and $s_{1}(t)$ and $s_{2}(t)$ are decomposed as shown in Figure 3. Signal $s_{1}(t)$ includes the data $a 1$ to $a 10$ in ten levels in the figure, and decomposed signal $s_{2}(t)$ includes the data 


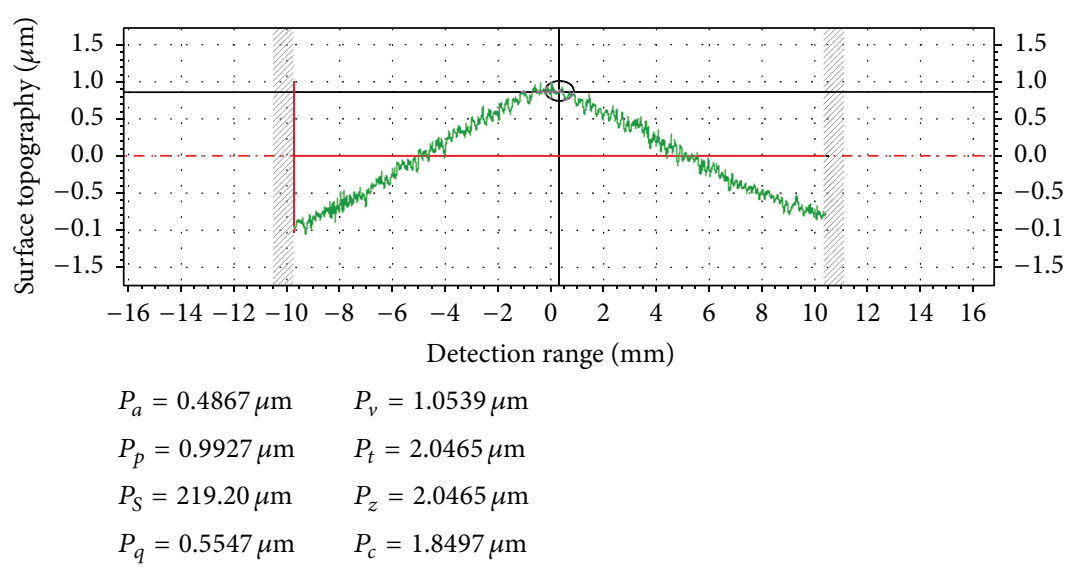

FIGURE 2: Surface topography of workpiece.

$d 1$ to $d 10$ in ten levels in the figure. The ordinate parameters $a 1-a 10$ represent a part of the signal with a low frequency, which belongs to signal $s_{1}(t)$ and corresponds to the static error of the machine tools. The ordinate parameters $d 1-d 10$ represent the part of signal with a high frequency, which belongs to the signal $s_{2}(t)$ and corresponds to the dynamic error of the machine tools. They correspond to the surface profile of the workpiece in the time domain. The actual signal in the horizontal coordinate is the data measured by the profile testing instrument. The number of the processed data is $10^{4}$, which is consistent with the test result in Figure 2; in Figure 3, the title of the horizontal coordinate is changed into the tool displacement; that is, the data number $10^{4}$ from the profile testing instrument is the same definition with the displacement $10 \mathrm{~mm}$ of the stylus in Figure 2.

The typical features of the processed signal with highfrequency should be extracted in the frequency field; the next part uses the spectral analysis method to identify the main error sources of the machine tool.

\section{Signal Evaluation in the Frequency Domain}

Power spectral density is usually used to analyze a signal in the frequency domain. It represents the change of signal power along with the frequency - that is, the distribution of the signal power in the frequency domain [22]. With the method, original description of the vibration in the time domain is transformed into the vibration description in the frequency domain. Because the analysis of the vibration is based on a large number of sample sets, the analysis obtained from a single sample is not accurate; thus, the statistical regularity of the vibration signal must be studied. The average power of the power signal $f(t)$ can be expressed as $P=$ $(1 / T) \int_{-T / 2}^{T / 2} f^{2}(t) d t$ in the time period $t \in[-T / 2, T / 2]$, if the signal $f(t)$ can be expressed as $f_{T}(t)$, and the Fourier transform of $f_{T}(t)$ is $F_{T}(\omega)=F\left[f_{T}(t)\right]$, where $F[]$ represents the Fourier transform. When the cycle $T$ increases, the energy of the $F_{T}(\omega)$ and $\left|F_{T}(\omega)\right|^{2}$ increases. When $T \rightarrow \infty, f_{T}(t) \rightarrow$ $f(t)$; at this point, $\left|F_{T}(\omega)\right|^{2} / T$ may become almost a limit.
If this limit exists, the average power can also be expressed in the frequency domain; that is,

$$
\begin{aligned}
P & =\lim _{T \rightarrow \infty} \frac{1}{T} \int_{-T / 2}^{T / 2} f^{2}(t) d t \\
& =\frac{1}{2 \pi} \int_{-\infty}^{\infty} \lim _{T \rightarrow \infty} \frac{\left|F_{T}(\omega)\right|^{2}}{T} d \omega,
\end{aligned}
$$

where $\left|F_{T}(\omega)\right|^{2} / T$ defines the power density function of signal $f(t)$.

The dynamic errors of the machine tool are superimposed together with other vibration errors in the time domain. It is difficult to identify the main feature of the dynamic error only in the time domain; thus, the error signal is transformed into the frequency domain by power spectral density. Figure 4(a) shows the power distribution of the high-frequency signal $d 6$ to $d 10$ levels in Figure 3(b). The amplitude of frequency $f 1$ $(1.85 \mathrm{~Hz})$ in level $d 10$ is the maximum, which is approximately 0.9 ; the amplitude of frequency $f 2(3.9 \mathrm{~Hz})$ in level $d 9$ is the second maximum, which is approximately 0.15 ; the amplitude of frequency $f 3(7.8 \mathrm{~Hz})$ in level $d 8$ is the third maximum, which is approximately 0.06 . Figure 4 (b) gives the power distribution of the high-frequency signal $d 1$ to $d 5$ levels in Figure 3(a). The amplitude of frequency $f 8(99 \mathrm{~Hz})$ is the maximum, which is approximately 0.034 ; the amplitude of frequency $f 9(149 \mathrm{~Hz})$ is the second maximum, which is approximately 0.15 ; the amplitude of frequency $f 3(7.8 \mathrm{~Hz})$ in level $d 8$ is the third maximum, which is approximately 0.0115 ; these frequencies represent the high energy of the signal, and they also indicate the main features of the error source of the machine tool.

\section{Error Source Identification in Time-Frequency Domain}

The surface topography of the workpiece includes static and dynamic errors of the machine tool. For the static error, such as the geometric errors, it belongs to the low-frequency signal, and it is difficult to identify the features in the frequency domain. Here it is identified in time domain in Section 4.3. 

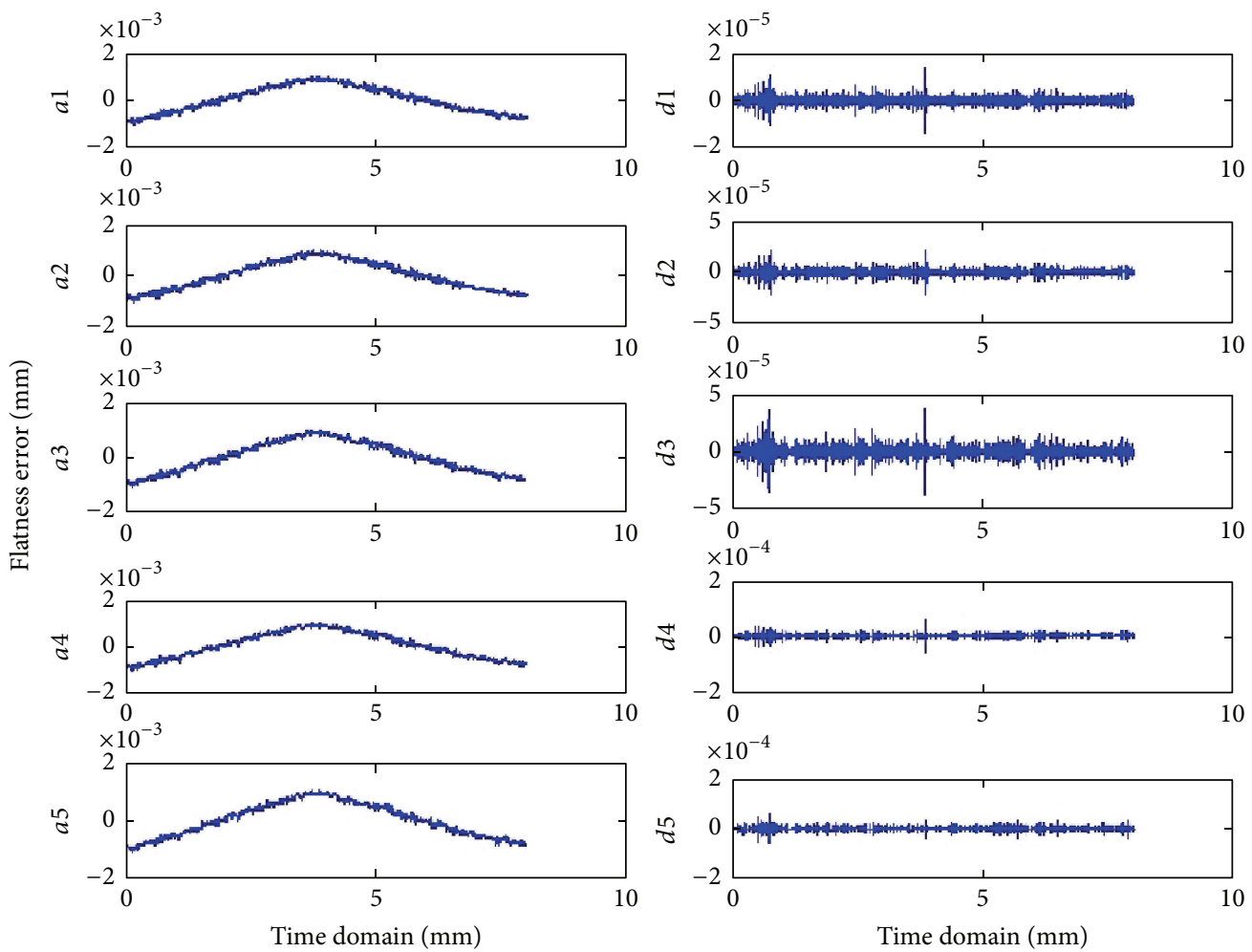

(a)
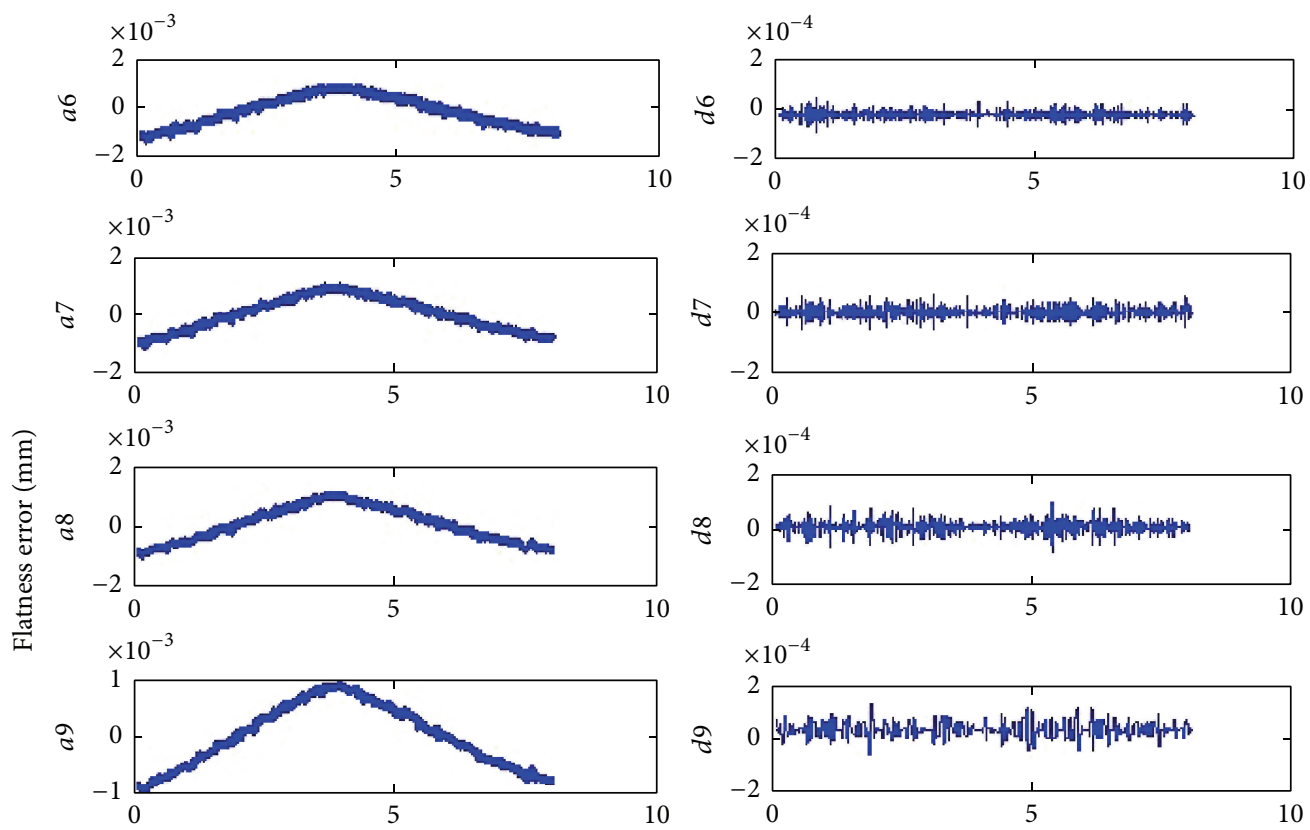

$\times 10^{-4}$
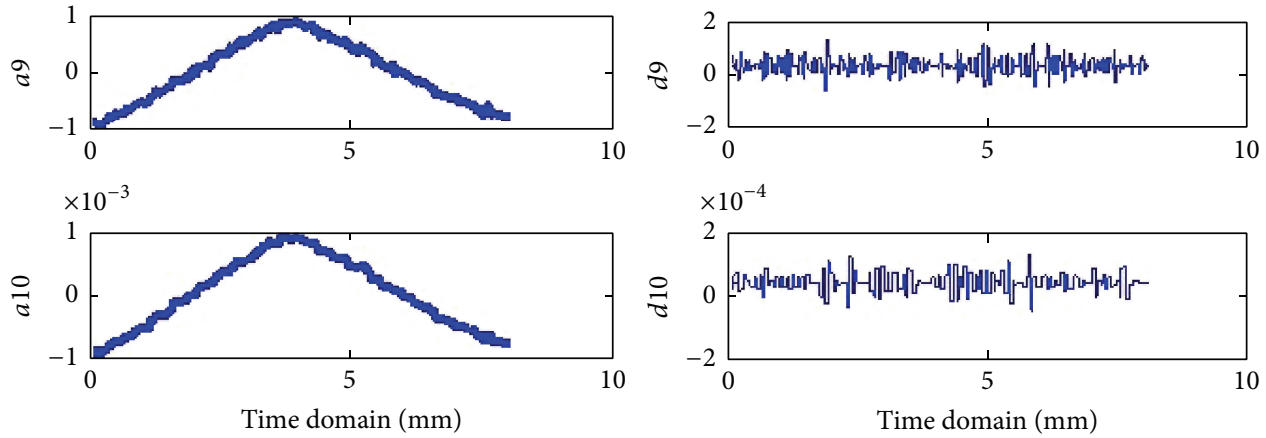

(b)

Figure 3: Processed result in ten levels. 

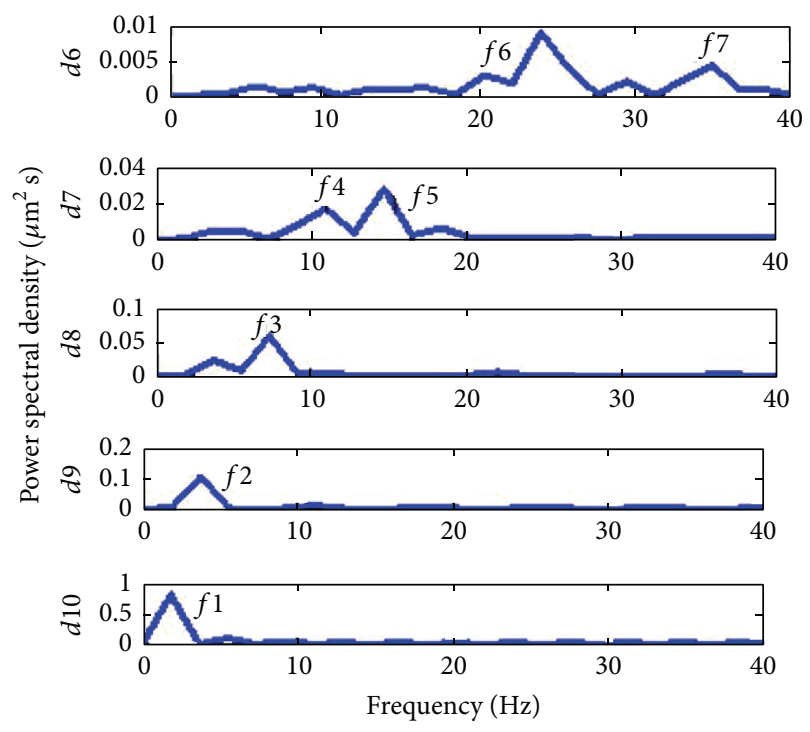

(a)

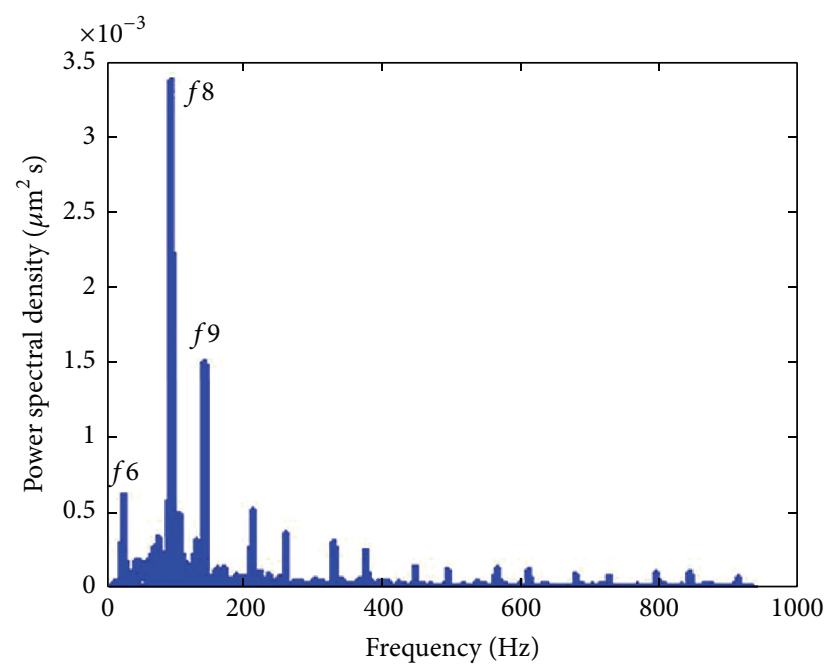

(b)

FIGURE 4: The analyzed result in frequency domain.

For dynamic errors, such as vibration signal, which includes the main spectral features of the key components of machine tools, this type of error should be identified in the frequency domain.

4.1. Extraction Imbalance Features of Spindle System in the Frequency Domain. When the spindle system is imbalanced, an excited force is generated in the process. The excited force has a relationship with the mass, the rotation speed, and the eccentricity of the spindle system. It continuously changes in the period, and the excited vibration of the spindle system will occur due to the excited force. When the frequency of the exciting force is close to the natural frequency of the spindle system, the resonance occurs. Therefore, the rotation frequency of the spindle system as the main spectral features will appear in the vibration signal. In the working process, the rotation speed of the spindle is $110 \mathrm{rpm}$, and the corresponding rotation frequency $110 / 60=1.83 \mathrm{~Hz}$ is obtained from the rotation speed. This rotation frequency or its multiple will appear in the vibration signal of the spindle system. From natural rotation frequency, the frequencies of the spindle system are obtained as shown in Table 1.

According to the above rotation frequencies of the spindle system, the surface topography of the workpiece in Figure 2 is analyzed. The prominent frequency in Figure 4 is evaluated; then the conclusion whether the high-frequency signal ( $d 1$ to $d 10$ in Figure 3) contains the corresponding frequency components of the spindle system should be determined. The corresponding frequency $f 1=1.85 \mathrm{~Hz}$ in $d 10$ in Figure 4(a) is similar to the rotation frequency $1.83 \mathrm{~Hz}$ of the spindle in Table 1 . This shows that the imbalanced signal of the spindle is present in the surface topology of the workpiece.

4.2. Spectrum Sign of Motor Imbalance and Interference Electrotherapy. Under normal circumstances, the operation
TABLE 1: Frequencies of the spindle system.

\begin{tabular}{lccc}
\hline \multicolumn{4}{c}{ Rotation frequency $(\mathrm{Hz})$} \\
\hline Natural & Twice & Triple & Quadruple \\
\hline 1.83 & 3.66 & 5.5 & 7.33 \\
\hline
\end{tabular}

time of the motor of the spindle system cannot be strictly controlled. Under some conditions, the motor is frequently initiated; thus, this will cause the shift of the balance block of the rotor, and finally the balance of the motor rotor is lost. Therefore, the vibration signal of the motor, including the eccentric harmonic, which is generated by the variation of the magnetic conduction wave of the rotor air gap, is caused by the mechanical dynamic eccentricity, and the corresponding frequency is [23]

$$
f_{\text {ecc }}=F_{1}\left(1+\left(\frac{1-s}{p / 2}\right)\right)=F_{1} \pm f_{r},
$$

where $F_{1}$ is the power frequency, $s$ is the slip ratio, $p$ is the series of the motor, and $f_{r}$ is the rotation frequency of the motor. In general, when the motor has a more serious eccentricity, the eccentric harmonic signal is also obvious. However, when the eccentricity is small, the composition of the eccentric harmonic is very small. The rotation speed and power frequency of the motor are known as $110 \mathrm{rpm}$ and $50 \mathrm{~Hz}$, and we can obtain the values of $f_{r}=1.84 \mathrm{~Hz}$ in the above equations. The two eccentric harmonics of the motor are obtained as $f_{\text {ecc- }}=48.16 \mathrm{~Hz}$ and $f_{\text {ecct }}=51.84 \mathrm{~Hz}$.

The frequency of the power frequency noise signal $f_{N}$ is usually between $49 \mathrm{~Hz}$ and $51 \mathrm{~Hz}$. However, in the rectification circuit, the frequency of the power frequency noise signal is an integer or subinteger multiple of $f_{N}$. In the result of Figure 4(a), the frequency of the peak spectral signal of $f 6$ in level $d 6$ is $25 \mathrm{~Hz}$, which is half the integer that determines 


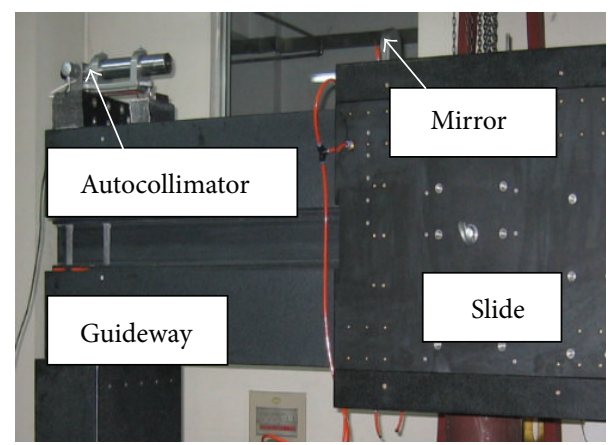

FIGURE 5: Experimental setup for measurement straightness of cross guideway.

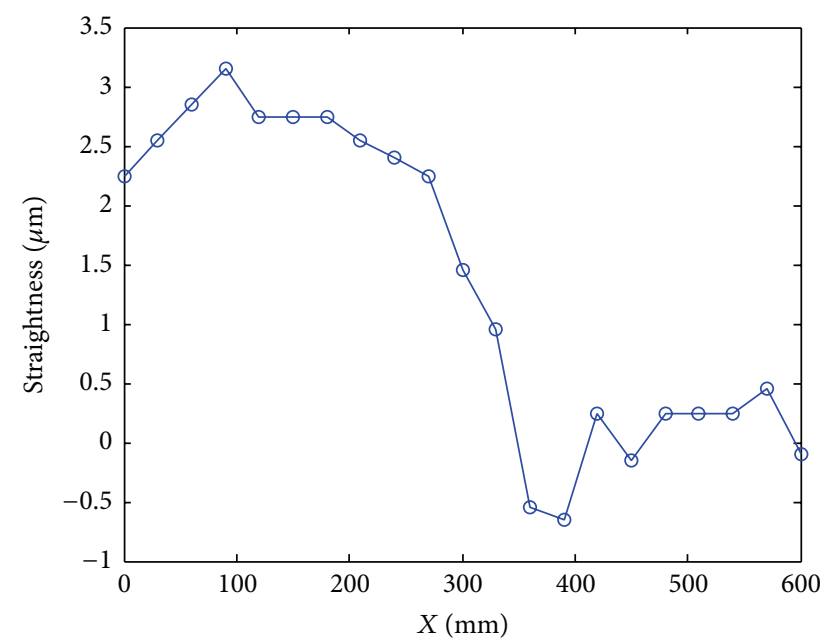

FIGURE 6: Measurement and fitting curve of straightness.

the high ordinate value $f 6$ at the coordinate $25 \mathrm{~Hz}$, which is the frequency of the subharmonic of $f_{N}$. This indicates that the AC interference exists in the surface topography of the workpiece.

\subsection{Identification Squareness of the Guideway from the Surface} Topography in the Time Domain. The main geometric error of the guideway is the straightness and squareness. Figure 5 shows the experimental setup for measuring the straightness of the cross guideway, which is the $X$ guideway with number 3 in Figure 1; it is an aerostatic guideway, including the fixed component (guideway) and the sliding component (slider). This provides the actual frame of the $X$ guideway of the processing lathe. The photoelectric autocollimator TA80 is mounted onto the cross guideway, and the mirror is mounted onto the cross slider. The measurement data are collected at $30 \mathrm{~mm}$ intervals, and the measured result is $0.3 \mu \mathrm{m} / 600 \mathrm{~mm}$. The result is shown in Figure 6. The abscissa shows the test range, from 0 to $600 \mathrm{~mm}$; the vertical coordinate is the value of straightness. To verify the influence of the errors of the guideway on the out-of-flatness of the workpiece, an aluminum workpiece is machined by the two-axis lathe, and the diameter of workpiece is $10 \mathrm{~mm}$.

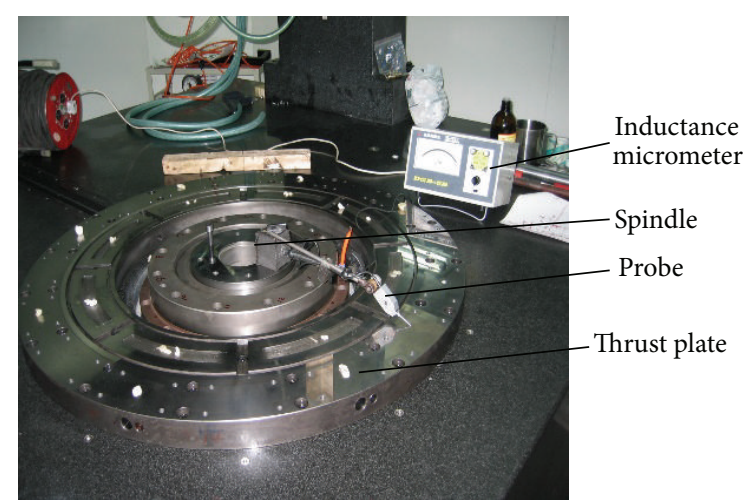

FIGURE 7: Measurement of squareness between guideway and spindle rotated line.

In order to identify the actual signal of the squareness error of guideway from the surface topography, the test of the squareness of the guideway is shown in Figure 7. An inductance micrometer is placed on the upper end face of the spindle, and the probe is in touch with the thrust plate (assuming that it is parallel with cross guideway) to measure the run-out of the spindle system. The probe rotates with the spindle together, the run-out of the thrust plate $r$ is obtained, and the largest deflection angle of the spindle can be deduced by $r$. It is assumed that the thrust plate is parallel with the $X$ guideway in the measurement; in fact, there is a parallelism error $p=2.5 \mathrm{~m}$ between them, which should be considered in the calculation for the deflection angle of the spindle. This is shown in (11), and the amplitude value of the deflection angle is $2.47 \times 10^{-6} \mathrm{rad}$ :

$$
\theta_{0} \approx \tan \left(\theta_{0}\right)=\frac{r+p}{L}
$$

The amplitude value of the squareness is $1.7 \mathrm{~mm}$, which can be derived from the above deflection angle. The squareness profile of the guideway is shown in Figure 8. This is very similar to the decomposed signal $a 1$ in Figure 3.

Here, the correlation analysis is used. The test process is $x(t)=s_{1}(t)+s_{2}(t)$, where $s_{1}(t)$ is the low-frequency signal of the surface topography decomposed by db1 WT, $s_{2}(t)$ is the corresponding high frequency, and the geometric error of the guideway is $y(t)=y_{1}(t)+y_{2}(t)$, where $y_{1}(t)$ is the squareness error of the guideway from the measured result in Figure 7, and $y_{2}(t)$ is the straightness error of the guideway from the measured result in Figure 5. The correlation between the low-frequency part of the surface topography $x_{1}(t)$ and squareness $y_{1}(t)$ and straightness $y_{2}(t)$ is analyzed; therefore the correlation coefficients $\gamma_{x y_{m}}$ and $\gamma_{x_{1} y_{m}}$ are obtained by equations as follows:

$$
\gamma_{x_{1} y_{m}}=\frac{\sum_{i=1}^{n}\left(x_{i}-\bar{x}\right)\left(y_{m i}-\overline{y_{m}}\right)}{\sqrt{\sum_{i=1}^{n}\left(x_{i}-\bar{x}\right)^{2}} \sqrt{\sum_{i=1}^{n}\left(y_{m i}-\overline{y_{m}}\right)^{2}}},
$$

where $m=1,2, \gamma_{x_{1} y_{m}}$ in (12) expresses the correlation coefficient between surface topographies $x_{1}(t)$ and $y_{1}(t)$ and $y_{2}(t)$. 


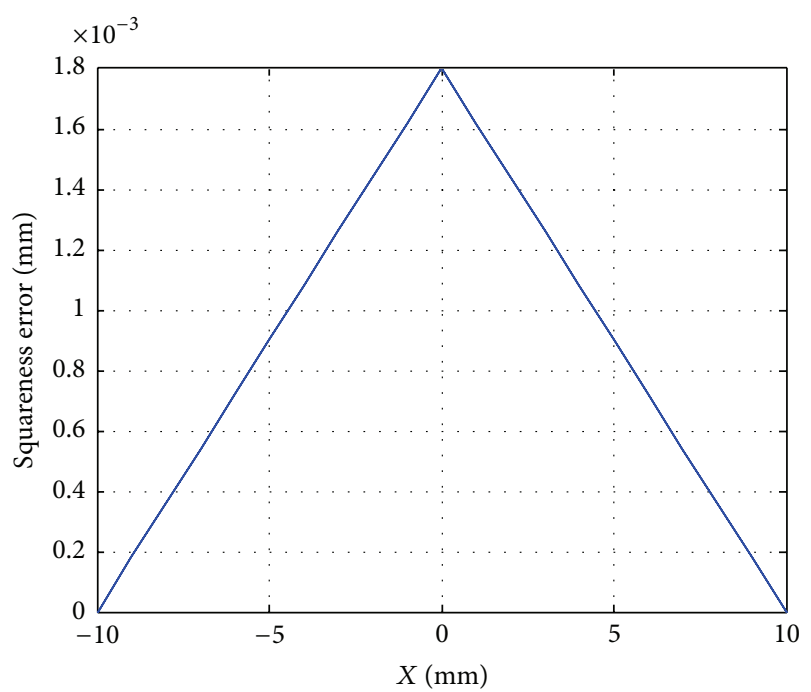

Figure 8: Squareness of guideway.

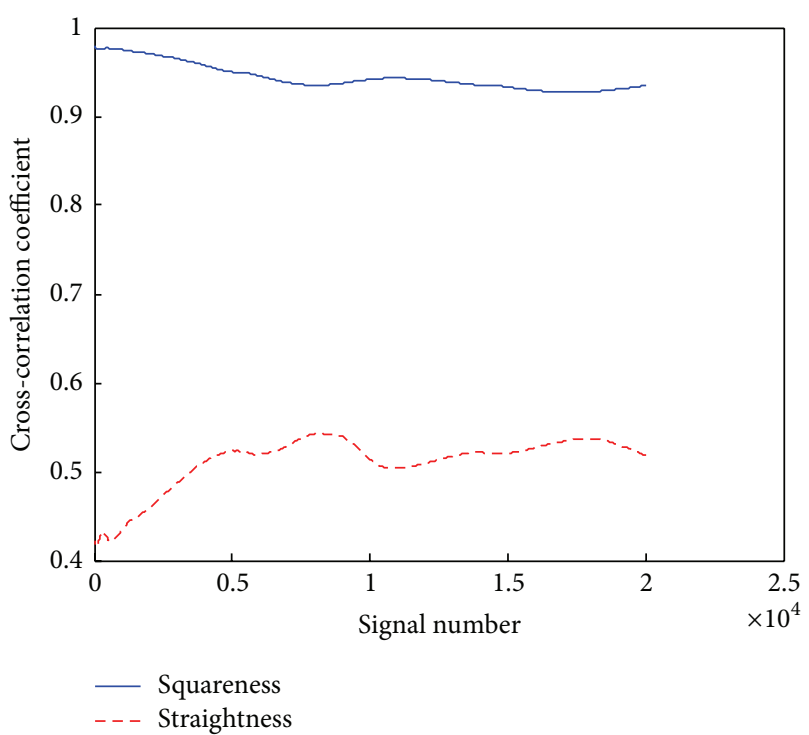

FIGURE 9: Cross-correlation analysis between surface topography and guideway error.

Figure 9 shows the results of the correlation analysis for the low-frequency part of the surface topography $x_{1}(t)$ processed by the wavelet transform. The correlation coefficient $\gamma_{x_{1} y_{1}}$ of the squareness error and the surface topography is close to 1, which means that the squareness error of the guideway is the main error in the test results and that the cross-correlation method is valid to identify the main error of the guideway. The correlation coefficient $\gamma_{x_{1} y_{2}}$ of the squareness error is approximately 0.5 , which is lower than the value of $\gamma_{x_{1} y_{1}}$. This indicates that the straightness is not the main error of the machining workpiece.

\section{Results and Discussion}

According to the frequency information for the rotary spindle, a spectrum analysis method is applied to extract the spectrum features of the high-frequency part of the surface topography signal processed by WT. With the measured geometric error of the guideway, the main geometric error (squareness) is identified from the low-frequency part of the surface topography signal. This identification method is a general method that is suitable for other types of machine tools. The measured vibration signal of the system is shown in Figures 10, and Figure 10(a) is the vibration result for the spindle system in the working condition; Figure 10(b) corresponds to the state in which only the air compressor is working; Figure 10(c) corresponds to the state in which the air compressor and oil pump are working; Figure $10(d)$ corresponds to the state in which the air compressor and electronic control system are working. From the figure, in all situations, the two frequencies $25 \mathrm{~Hz}$ and $33 \mathrm{~Hz}$ still exist. These two values are consistent with the above $f 6=25 \mathrm{~Hz}$ and $f 7=35 \mathrm{~Hz}$ in Figure 4(a), which shows the correctness and effectiveness of the above signal process.

The value of $f 1(1.85 \mathrm{~Hz}$ in level $d 10$ in Figure $4(\mathrm{a}))$ relates to the natural rotation frequency $(1.83 \mathrm{~Hz}$ in Table 1$)$ of the spindle system, and its corresponding amplitude is the maximum amplitude in level $d 10$ in the Figure 4(a), and this also represents the high energy.

The frequency $f 6(25 \mathrm{~Hz}$ in level $d 6$ in Figure $4(\mathrm{a}))$ has the peak spectral amplitude, and it is the half of the power frequency of the motor; this indicates that the $\mathrm{AC}$ interference exists in the surface topography of the workpiece. The motor is connected with the shaft in the spindle system of the machine tools; thus, the imbalance of the spindle is the main error source of the machine tool.

The cross-correlation coefficient of squareness and the surface topography is approximately 0.98 , which is higher than the value of straightness 0.55 in Figure 9; this indicates that the main error of the low signal $a 1$ in Figure 3 is the squareness error of the guideway.

\section{Conclusions}

The investigation, which considers the error sources of machine tools, provides an analytical relationship between the surface topography and the guideway geometric errors, which involve the imbalance of the spindle system. On the one hand, with the corresponding frequency information of the rotary spindle, this method can extract the main features of the imbalance of the spindle system from the surface topography of the workpiece in the frequency domain; on the other hand, with the correlation coefficient between the guideway errors and surface topography of the workpiece, it can identify the main geometric error of guideway in the time domain. With wavelet analysis, the identification precision of the test signal is improved, and the dominant error sources of the machine tool that impact the surface topography of the workpiece are identified. The method can also be applied to the error identification of other components of the machine tool. 


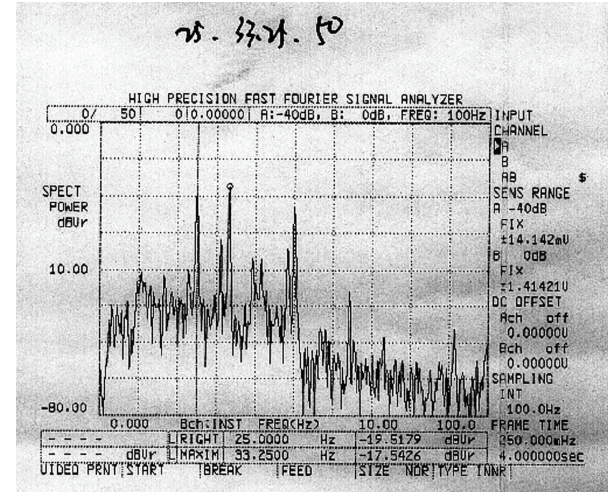

(a)

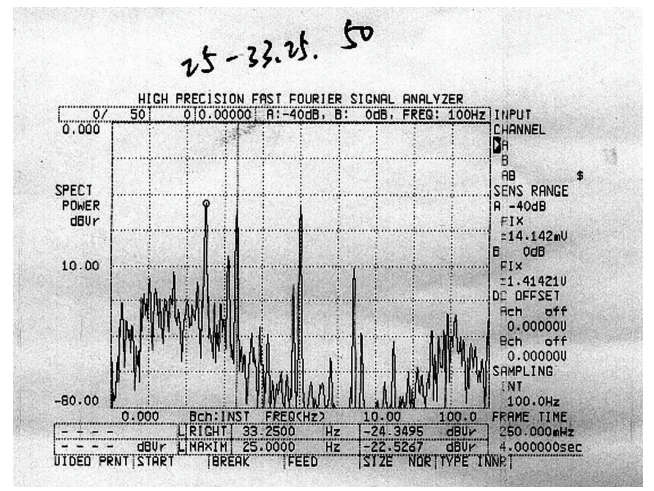

(c)

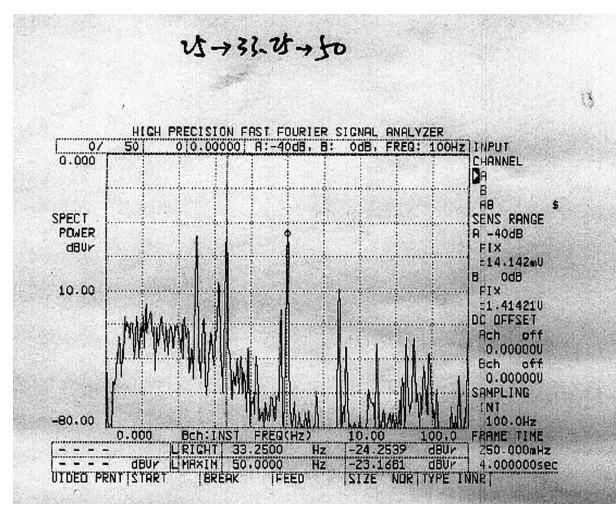

(b)

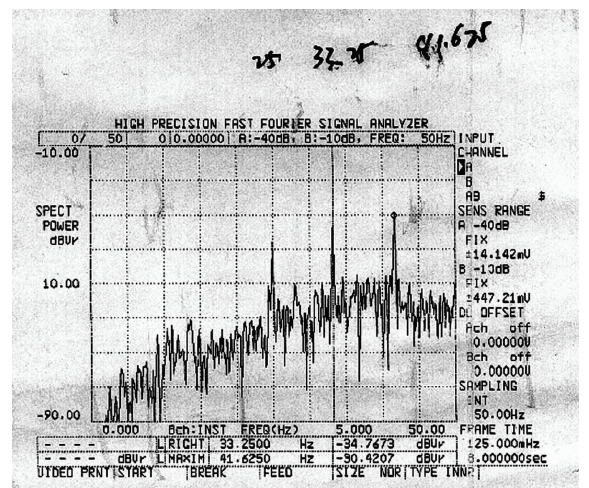

(d)

FIGURE 10: Measured signal of spindle system in different conditions. (a) Case is turning on all, that is, the air pump, the oil pump, and the electronic control system; (b) case is just turning on the air pump; (c) case is turning on the air pump and oil pump; (d) case is turning on the air pump and electronic control system.

\section{Conflict of Interests}

The authors declare no conflict of interests.

\section{Authors' Contribution}

Dongju Chen and Shuai Zhou conceived and designed the study. Dongju Chen and Jinwei Fan performed the experiments. Dongju Chen and Lihua Dong wrote the paper. All authors read and approved the paper.

\section{Acknowledgments}

This research was funded by the National Natural Science Foundation of China (Grants nos. 51105005 and 51475010) and the Natural Science Foundation of Beijing Municipality (Grant no. 3142005).

\section{References}

[1] R. Ramesh, M. A. Mannan, and A. N. Poo, "Error compensation in machine tools-a review: part I: geometric, cutting-force induced and fixture-dependent errors," International Journal of Machine Tools and Manufacture, vol. 40, no. 9, pp. 1235-1256, 2000.
[2] T. L. Schmitz, M. A. Davies, and M. D. Kennedy, “Tool point frequency response prediction for high-speed machining by RCSA," Journal of Manufacturing Science and EngineeringTransactions of the ASME, vol. 123, no. 4, pp. 700-707, 2001.

[3] Z. K. Peng, P. W. Tse, and F. L. Chu, "A comparison study of improved Hilbert-Huang transform and wavelet transform: application to fault diagnosis for rolling bearing," Mechanical Systems and Signal Processing, vol. 19, no. 5, pp. 974-988, 2005.

[4] C. Quan, H. Niu, and C. J. Tay, "An improved windowed Fourier transform for fringe demodulation," Optics and Laser Technology, vol. 42, no. 1, pp. 126-131, 2010.

[5] L. Durak, "Shift-invariance of short-time fourier transform in fractional fourier domains," Journal of the Franklin Institute, vol. 346, no. 2, pp. 136-146, 2009.

[6] H. S. Li, S. Wu, and H. Kratz, "FFT and wavelet-based analysis of the influence of machine vibrations on hard turned surface topographies," Tsinghua Science and Technology, vol. 12, no. 4, pp. 441-446, 2007.

[7] K. Lingadurai and M. S. Shunmugam, "Metrological characteristics of wavelet filter used for engineering surfaces," Measurement, vol. 39, no. 7, pp. 575-584, 2006.

[8] J. Antonino-Daviu, P. Jover, M. Riera, A. Arkkio, and J. RogerFolch, "DWT analysis of numerical and experimental data for the diagnosis of dynamic eccentricities in induction motors," Mechanical Systems and Signal Processing, vol. 21, no. 6, pp. 2575-2589, 2007. 
[9] S. Chand, "SC- and SS-wavelet transforms," Signal Processing, vol. 89, no. 3, pp. 305-313, 2009.

[10] K. P. Zhu, Y. S. Wong, and G. S. Hong, "Wavelet analysis of sensor signals for tool condition monitoring: a review and some new results," International Journal of Machine Tools and Manufacture, vol. 49, no. 7-8, pp. 537-553, 2009.

[11] P. S. Bhowmik, P. Purkait, and K. Bhattacharya, "A novel wavelet transform aided neural network based transmission line fault analysis method," International Journal of Electrical Power \& Energy Systems, vol. 31, no. 5, pp. 213-219, 2009.

[12] M. H. A. Bonte, A. de Boer, and R. Liebregts, "Determining the von Mises stress power spectral density for frequency domain fatigue analysis including out-of-phase stress components," Journal of Sound and Vibration, vol. 302, no. 1-2, pp. 379-386, 2007.

[13] K. Yoshida, "Power spectral density peak estimation from broadband data," Journal of Sound and Vibration, vol. 312, no. 4-5, pp. 893-905, 2008.

[14] F. Lu, D. Kennedy, F. W. Williams, and J. H. Lin, "Symplectic analysis of vertical random vibration for coupled vehicle-track systems," Journal of Sound and Vibration, vol. 317, no. 1-2, pp. 236-249, 2008.

[15] H. Schulz and T. Würz, "Balancing requirements for fast rotating tools and spindle systems," CIRP Annals-Manufacturing Technology, vol. 47, no. 1, pp. 321-324, 1998.

[16] S. Jiang and S. Zheng, "A modeling approach for analysis and improvement of spindle-drawbar-bearing assembly dynamics," International Journal of Machine Tools and Manufacture, vol. 50, no. 1, pp. 131-142, 2010.

[17] L. Ziran, W. Jinxuan, and H. Tao, "Application of adaptive filtering based on harmonic wavelet in the dynamic unbalance of electro-spindle," in International Conference on Measuring Technology and Mechatronics Automation (ICMTMA '10), pp. 597-600, Changsha City, China, March 2010.

[18] C. Quan, H. Niu, and C. J. Tay, "An improved windowed Fourier transform for fringe demodulation," Optics \& Laser Technology, vol. 42, no. 1, pp. 126-131, 2010.

[19] L. Durak, "Shift-invariance of short-time Fourier transform in fractional Fourier domains," Journal of the Franklin Institute, vol. 346, no. 2, pp. 136-146, 2009.

[20] W. Wieleba, "The statistical correlation of the coefficient of friction and wear rate of PTFE composites with steel counterface roughness and hardness," Wear, vol. 252, no. 9-10, pp. 719-729, 2002.

[21] W. D. Lockwood and A. P. Reynolds, "Use and verification of digital image correlation for automated 3-D surface characterization in the scanning electron microscope," Materials Characterization, vol. 42, no. 2, pp. 123-134, 1999.

[22] R. N. Youngworth, B. B. Gallagher, and B. L. Stamper, "An overview of power spectral density (PSD) calculations," in Optical Manufacturing and Testing VI, vol. 5869 of Proceedings of SPIE, August 2005.

[23] K. D. Hurst and T. G. Habetler, "Sensorless speed measurement using current harmonic spectral estimation in induction machine drives," IEEE Transactions on Power Electronics, vol. 11, no. 1, pp. 66-73, 1996. 


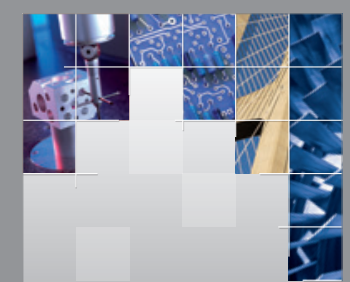

\section{Enfincering}
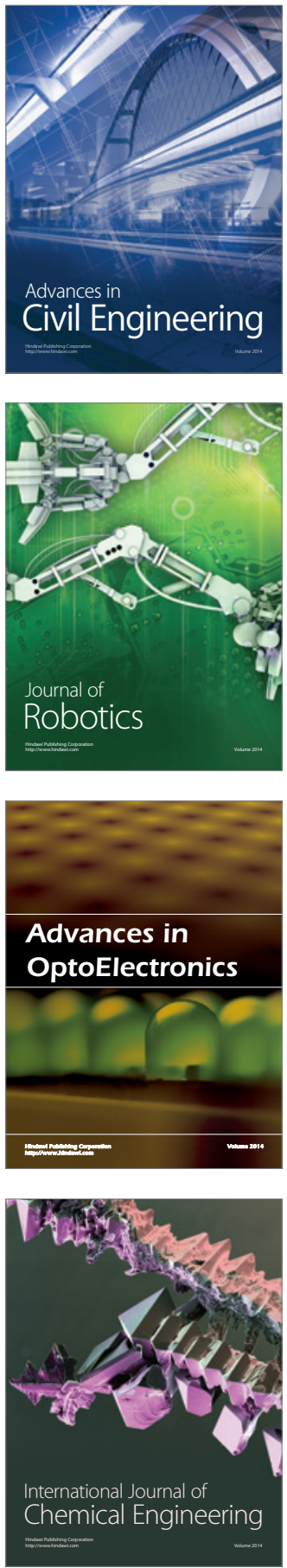

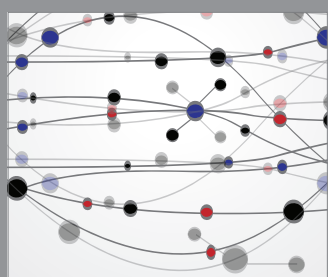

The Scientific World Journal

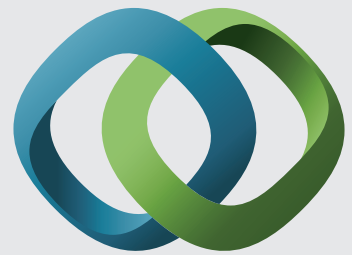

\section{Hindawi}

Submit your manuscripts at

http://www.hindawi.com
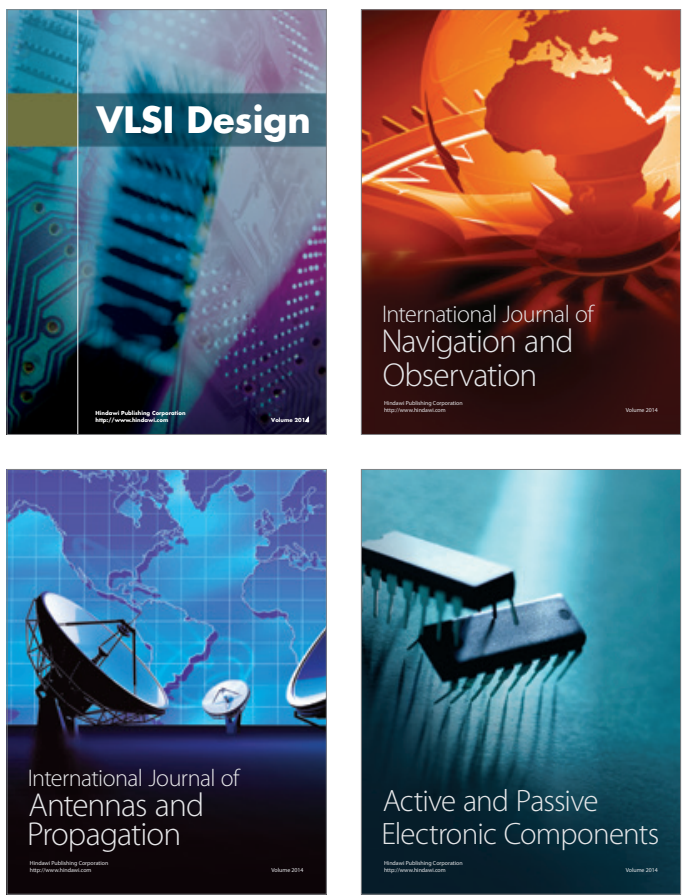
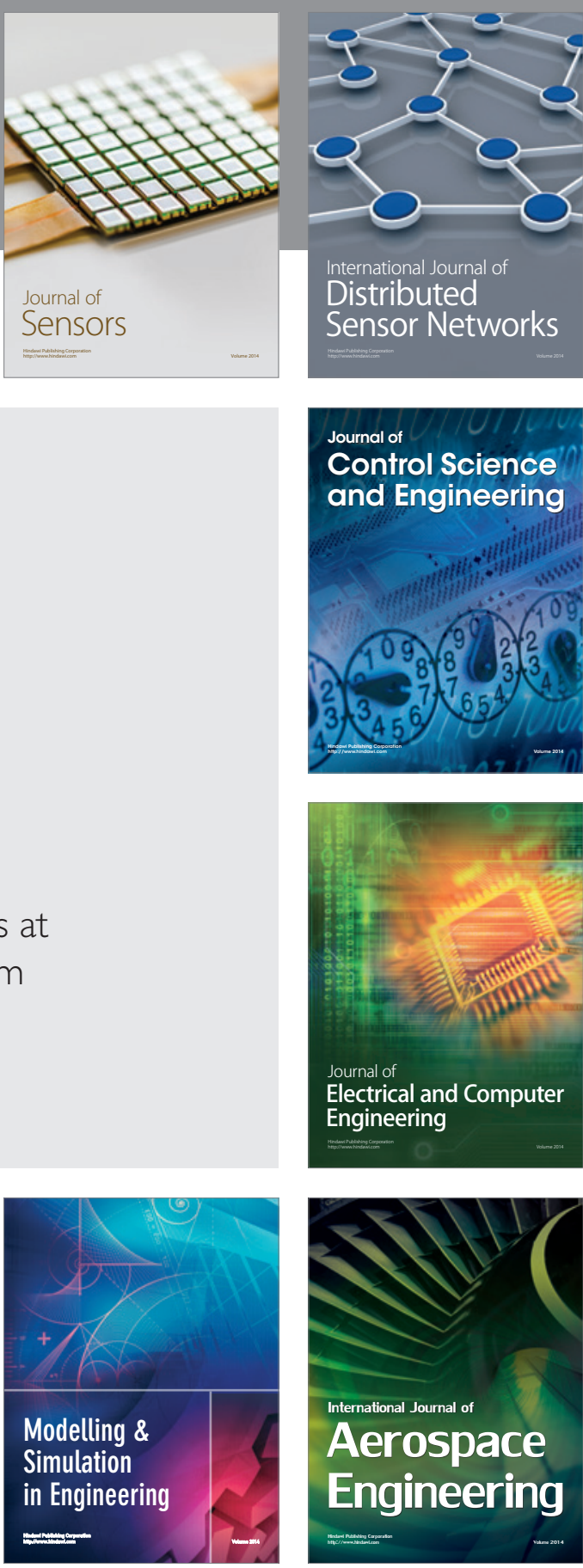

International Journal of

Distributed

Sensor Networks

Journal of

Control Science

and Engineering
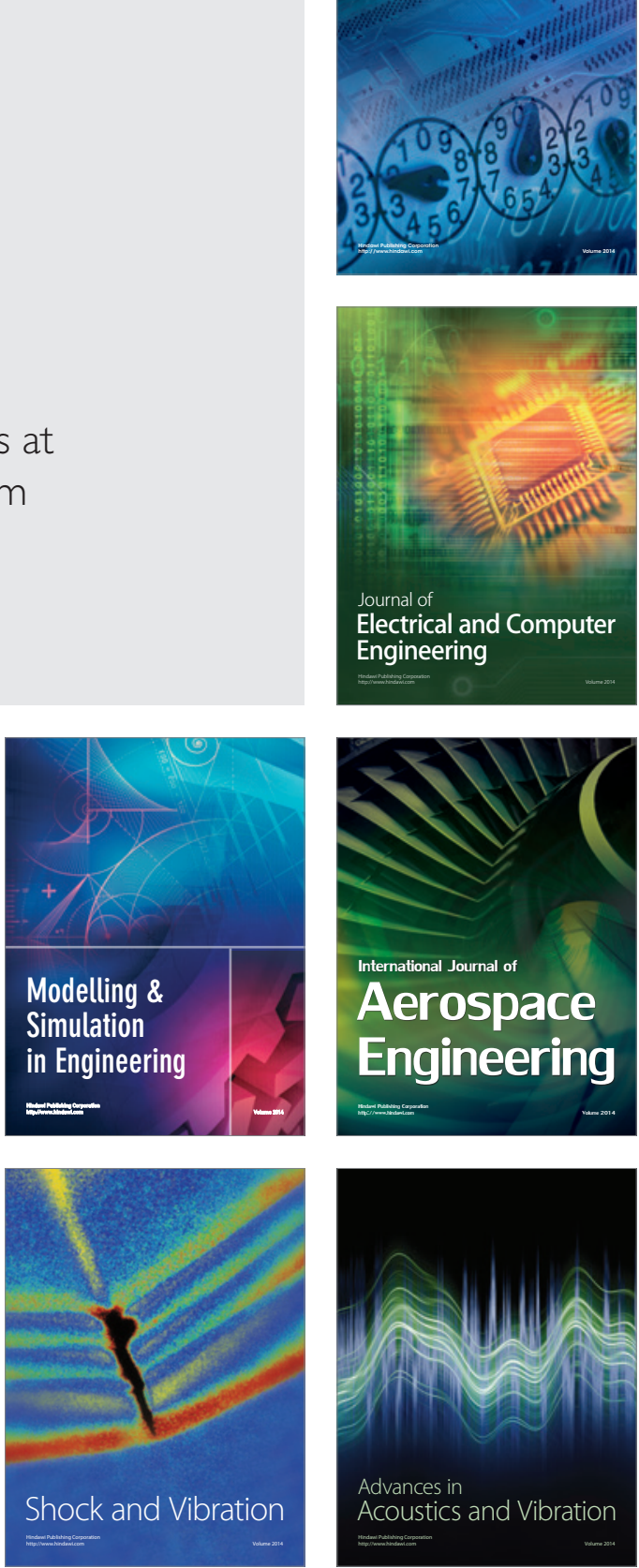